\title{
LA INFLUENCIA FUTURA DE LA PANDEMIA DESDE EL PLANO PSICOLOGICO EN LOS ADOLESCENTES
}

\author{
Álvaro Muelas Plaza \\ Universidad Villanueva (Madrid) \\ alvaro_muelas@hotmail.com
}

Recepción Artículo: 13 octubre 2021 Admisión Evaluación: 13 octubre 2021 Informe Evaluador 1: 13 octubre 2021 Informe Evaluador 2: 14 octubre 2021 Aprobación Publicación: 14 octubre 2021

\section{RESUMEN}

Los primeros meses del año 2020 se recordarán en todo el mundo como uno de los momentos más importantes en la historia del mundo, debido a la aparición del COVID-19, que produjo que en apenas unos días el mundo se paralizara. El 11 de marzo de 2020 la Organización Mundial de la Salud (OMS) tuvo que declarar la COVID-19 como pandemia, haciendo que tres días más tarde, el 14 de marzo de 2020 el Gobierno de España tuviera que aprobar un Real Decreto (RD 463/2020, declarando un Estado de alarma, con el objetivo de proteger de la mejor manera posible a toda la ciudadanía y evitar que la enfermedad se pudiera propagar rápidamente por toda la población. Esta circunstancia ha hecho que desde ese momento la vida de cualquier ciudadano ha cambiado desde todos los ámbitos de su vida, y uno de ellos es de donde este artículo se quiere centrar, como es desde el plano psicológico. Los hechos cotidianos integrados en el día a día de una persona se han visto afectados, y si en cualquier edad es importante, más lo es en etapas evolutivas como la adolescencia, en donde la persona se encuentra en un momento vital donde necesita de una manera notoria su búsqueda de identidad. Se ha visto a través de estudios recientes (Orgiles, et.al, 2020) como la pandemia ha influido en el aumento de estrés y ansiedad en los adolescentes, al igual que en su estado emocional, sintiéndose enfadados, nerviosos, irascibles y tristes (Tiwari et al., 2020). A través de este artículo se pretende dar respuesta a las necesidades psicológicas futuras que van a necesitar los adolescentes en su desarrollo personal.

Palabras clave: pandemia; estado de alarma; adolescencia; estado emocional; necesidades psicológicas

\section{ABSTRACT}

The future influence of the pandemic from the psychological level on adolescents. The first months of 2020 will be remembered around the world as one of the most important moments in the history of the world, due to the appearance of COVID-19, which caused the world to come to a standstill in just a few days. On March 11, 2020, the World Health Organization (WHO) had to declare COVID-19 a pandemic, causing three days later, on March 14, 2020, the Government of Spain had to approve a Royal Decree (RD 463/2020, declaring a state of alarm, with the aim of protecting the entire citizenry in the best possible way and preventing the disease from 


\section{LA INFLUENCIA FUTURA DE LA PANDEMIA DESDE EL PLANO PSICOLOGICO EN LOS ADOLESCENTES}

spreading rapidly throughout the population. This circumstance has made the life of any Citizen has changed from all areas of his life, and one of them is where this article wants to focus, as it is from the psychological plane. The daily events integrated into the day-to-day life of a person have been affected, and if At any age it is important, more so in evolutionary stages such as adolescence, where the person is in a vital moment where they clearly need their search for identity. It has been seen through recent studies (Orgiles, et.al, 2020) as the pandemic has influenced the increase in stress and anxiety in adolescents, as well as their emotional state, feeling angry, nervous, irascible and sad (Tiwari et al., 2020). Through this article it is intended to respond to the future psychological needs that adolescents will need in their personal development.

Keywords: pandemic; state of alarm; adolescence; emotional state; psychological needs

\section{ÁREA TEMÁTICA. ANTECEDENTES DE LA TEMÁTICA A TRATAR}

Los últimos meses del año 2019 y los primeros del 2020, se recordarán especialmente en todo el mundo en las futuras generaciones, debido a la aparición del SARS-CoV-2, virus que produce el COVID-19, enfermedad infecciosa que está produciendo muchas muertes y significativas secuelas en la población de todo el mundo. El 11 de marzo de 2020 la Organización Mundial de la Salud (OMS) tuvo que declarar la COVID-19 como pandemia, haciendo que tres días más tarde, el 14 de marzo de 2020 el Gobierno de España tuviera que aprobar un Real Decreto (RD 463/2020, declarando un Estado de alarma, con el objetivo de proteger de la mejor manera posible a toda la ciudadanía y evitar quela enfermedad se pudiera propagar rápidamente por toda la población.

Según la RAE (2021) un estado de alarma es una situación extraordinaria que el Consejo de Ministros de un país declara a causa de catástrofes, crisis sanitarias, etc., dando lugar a importantes alteraciones en el desarrollo del día a día de la ciudadanía de un país.

Desde principios del año 2020, el COVID-19 ha hecho que todo haya cambiado, afectando a todos los estamentos y sectores sociales. En relación al tema que nos afecta en este artículo, desde el plano psicológico, dicha pandemia está teniendo secuelas muy notorias en toda la población, y lo que es más importante, va a ser un tema muy importante a tener en cuenta en los próximos años, debido a que todos los hábitos y costumbres de las personas se han visto afectadas.

En la actualidad, la propagación del virus está haciendo que para evitar en la medida de lo posible su máxima expansión, las personas afectadas tienen que estar confinadas durante el periodo indicado por las autoridades sanitarias, haciendo que se produzca una situación de aislamiento temporal, y lo más notorio, unos efectos negativos en todos los planos de una persona, destacando el psicológico. Dicho confinamiento, en relación a los adolescentes, aunque en principio fue aceptado de muy buena manera, por el hecho de no acudir a clase y estar en casa, el paso de los días y las semanas alteró en todos los aspectos su rutina, que si es importante en cualquier etapa de la vida, lo es más en la adolescencia, teniendo un impacto muy notorio en su salud somática y emocional. (Balluerka, et. al., 2020). Desde el plano somático, al estar tanto tiempo en casa y así reducir el movimiento y el ejercicio físico y ocupar dicho tiempo con herramientas tecnológicas, ha habido una importante tendencia a la obesidad, favorecido también por una alimentación procesada y rica en hidratos de carbono, al igual que está produciendo problemas de sueño. Desde el plano emocional, el estrés producido por la nueva situación, relacionada por a la incertidumbre vivida, ha desencadenado en cuadros de malestar afectivo-emocional, acompañados de desasosiego y ansiedad por desconocer su futuro próximo. Los adolescentes ante esta situación se sienten incómodos en su entorno, haciendo que la convivencia con ellos, por la pérdida de "libertad" sea en ocasiones complicada.

Según el primer estudio que se realizó en China, para analizar el impacto psicológico del COVID-109 en su población, se observó como los dos factores que más influyen tanto en el bienestar psicológico y físico son la pérdida de los hábitos diarios de una persona (dando lugar a cambios negativos en las relaciones sociales, en la alimentación, en el sueño, etc.) y el estrés psicosocial que esta situación produce (Wang, Pan et al., 2020). El ser humano estamos hechos para vivir en sociedad y con gente; de la noche a la mañana, esto se ha perdido, afec- 
tando al plano emocional y psicológico. El ser humano, psicológicamente necesita conocer lo mejor posible su entorno; si no es así, esto hace que se produzcan cuadros importantes de estrés, manifestándose en situaciones como miedo al virus y sentimientos de incertidumbre, por no tener información contrastada científicamente (Brooks et al., 2020), afectando de un modo muy notorio al plano psicológico.

En relación a la población española (Orgiles, et.al, 2020) dentro de los primeros resultados conocidos en relación a los efectos psicológicos del COVID 19, se puede observar como el 75,7\% de las personas atendidas psicológicamente lo tuvieron que hacer por estrés y ansiedad y el 56,2\% por sintomatología depresiva. El 45,7\% de las personas pertenecientes a la muestra del estudio realizado, indicaron que había aumentado su malestar psicológico, y el nivel de incertidumbre ante la situación que se estaba viviendo, había aumentado en el 77,5\% de los casos, afectando en aspectos tan concretos como el sueño en el 52,8\%.

Otro de los aspectos a tener en cuenta ocasionado por la pandemia (Orgiles, et.al, 2020), es que el malestar psicológico afecta más en la población de menor edad, siendo en edades comprendidas entre 18 y 3 años del $53 \%$; entre 35 y 60 años, del 44\%, y en mayores de 60 años del 34\%, estos resultados van acompañados de problemas de concentración, donde se pudo apreciar como en I población más joven, el grado de incidencia era mayor, siendo del $53 \%$ entre 18 y 34 años; del $41 \%$ entre los 35 y 60 años y del 25\% en los mayores de 60.

Siguiendo la línea de los efectos psicológicos producidos por el COVID-19 en los adolescentes, se aprecia como los síntomas emocionales y los problemas de conducta han sido las más afectadas. Teniendo en cuenta la relación entre jóvenes y sus familias, estos últimos han manifestado en diversos estudios que sus hijos en el $70 \%$ de los casos se han encontrado más nerviosos y enfadados (Orgilés et al., 2020; Tiwari et al., 2020); e irascibles y tristes (Berasategui Sancho et al., 2020). Otro de los aspectos a tener en cuenta es que durante el confinamiento, los adolescentes han tenido miedo, traducido en miedo a contagiarse, a que sus familiares cercanos se pudieran contagiar, a contagiar a otras personas, y fundamentalmente han manifestado el miedo y la incertidumbre a lo qué pasaría al poder hacer actividades atractivas, y según ellos, necesarias para ellos, debido a que eso les ha producido una importante sentimiento de soledad (Berasategui et al., 2020; Orgilés et al., 2020).

El sentimiento de miedo es un aspecto muy a tener en cuenta, fundamentalmente en estos momentos, donde la evolución del COVID-19 se desconoce, debido a que estudios anteriores has demostrado cientííicamente la clara relación existente entre el miedo y la predisposición a padecer trastornos como la ansiedad y la depresión (Oppedal e Idsoe, 2012; Rodríguez- Hernández et al., 2014; Sandín et al., 1998; Valiente et al., 2003), o trastornos obsesivos-compulsivos en relación a miedos aparecen por el desconocimiento de los virus (Arnáez et al., 2015),y en concreto por la incertidumbre que esta produciendo en la sociedad, al igual que la preocupación que existe por el contagio, debido a que realmente en un procentaje alto no se conoce cuando existe mayor o menos probabildad de ello y por la pérdida de los seres queridos (Balluerka-Lasa et al., 2020).

Ante la situación de incertidumbre y miedo que existe actualmente en la población en relación al COVID 19, y aunque las causas y consecuencias de problemas mentales son muy difíciles y nada convenientes de generalizar, ya que depende de muchísimos factores (herencia genética, edad, estado físico, acontecimientos vividos personalmente, los medios de comunicación, etc.), sería muy aconsejable conocer los miedos existente, no solo para prevenirlos, sino para evitar actitudes discriminatorias hacia los infectados o persona que se puede pensar que lo pueden estar (Valiente et al.,2020). En recientes estudios, se ha comprobado como a mayor tiempo de confinamiento, el estado psicológico de los adolescentes empeora, aumentando losmiedos hacia el distanciamiento social, la contaminación con otras personas o el impacto en la vida (Brooks et al.,2020)

Teniendo en cuenta los datos indicados, y tras unos meses de confinamiento y de enorme incertidumbre en todos los aspectos de la vida de una persona, toca el momento de intentar paulatinamente volver a la "normalidad" que teníamos antes de la pandemia. Para ello, es de vital importancia la infomación científica, para que esto pueda producir en la sociedad, y de un modo prioritario en los adolescentes, lo máximo posible seguridad (Sandín, et. al., 2020) y confianza en ellos y en su alrededor. A través de esta circunstancia, y desde la construcción de valores (como el respeto, la solidaridad, el esfuerzo, la comprensión, etc....), se podrá construir una 


\section{LA INFLUENCIA FUTURA DE LA PANDEMIA DESDE EL PLANO PSICOLOGICO EN LOS ADOLESCENTES}

sociedad, en donde la pandemia haya valido para formar mejores personas y una mejor sociedad, donde todos sus integrantes podamos remar en una dirección para conseguir un objetivo común (Green, 2020).

Ante la situación social existente en la actualidad, trabajar desde la prevención de problemas psicológicos en la población es un aspecto que se debe considerar prioritario, centrándose en las siguientes líneas de trabajo (Espada, et. al., 2020): 1) Dar a conocer a la población de un modo científico el COVID-19; 2) Informar a la población, con datos contrastados científicamente de la situación real de la pandemia.3) Dar a conocer medidas preventivas; 4) Elaborar planes de actuación a partir de medidas preventivas; 5) Informar de comportamientos personales que puedan ser señales de ayuda psicológica por parte de profesionales; y 6)Realizar estudios psicológicos, para conocer la evolución que tiene una persona en su tratamiento.

En definitiva, estamos ante un escenario en donde es de vital importancia que la población, y en especial los adolescentes, se encuentren bien en todos los ámbitos de su vida, pero en especial en el plano psicológico, debido a que serán muchas las incertidumbres y vicisitudes a las que se tendrán que enfrentar, y para ello un estado emocional estable y una autoconfianza plena harán que puedan salir con mayor garantías de la situación.

\section{OBJETIVOS DE LA INVESTIGACIÓN}

Teniendo en cuenta el marco teórico expuesto, el objetivo principal propuesto en esta investigación es:

Conocer la influencia de la pandemia desde el plano psicológico en los adolescentes.

Partiendo del citado objetivo, los objetivos específicos establecidos son los siguientes:

Conocer si por causa del confinamiento los adolescentes han aumentado el peso.

Saber si por causa de la situación vivida los adolescentes se encuentran más nerviosos.

Conocer si la pandemia a afectado en el sueño de los adolescentes.

Comprobar si el confinamiento ha afectado en el nivel de concentración de los adolescentes.

Saber si la pandemia les produce intranquilidad y preocupación a la hora de que se contagien tanto sus familiares como ellos.

\section{MUESTRA Y PARTICIPANTES}

Para dar respuesta a los objetivos establecidos en esta comunicación, se han seleccionado a 34 estudiantes de bachillerato de una clase, del itinerario de Ciencias Sociales, que cursan la asignatura de Psicología pertenecientes a un centro de Bachillerato de la Comunidad de Madrid.

\section{METODOLOGíA Y/O INSTRUMENTOS UTILIZADOS}

Para conocer la influencia de la pandemia desde el plano psicológico en los adolescentes, y así cumplir con el objetivo establecido en esta comunicación, se ha llevado a cabo una Investigación cuantitativa, descriptiva, y para ello, a comienzo del curso 21-22, en el mes de septiembre del 2021,como docente de la asignatura de psicología en el curso de $2^{\circ}$ de bachillerato, se realizó un cuestionario de elaboración propia con las siguientes preguntas y opción de respuesta:

Tabla 1. Cuestionario para conocer estado psicológico de adolescentes tras la pandemia

\begin{tabular}{|c|c|c|}
\hline & \multicolumn{2}{|c|}{ Respuesta } \\
\hline & SI & NO \\
\hline 1. Ha aumentado tu peso durante el confinamiento & & \\
\hline 2. Notas que estás más nervioso que antes del confinamiento & & \\
\hline 3. Duermes peor que antes del confinamiento & & \\
\hline $\begin{array}{l}\text { 4. A la hora de realizar alguna actividad, te cuesta más concentrarte } \\
\text { que antes de la pandemia }\end{array}$ & & \\
\hline 5. Te produce intranquilidad que tus familiares se puedan contagiar & & \\
\hline 6. Tienes preocupación a la hora de poder contagiarte & & \\
\hline
\end{tabular}

Fuente: Elaboración Propia. 


\section{RESULTADOS ALCANZADOS}

Partiendo del cuestionario cumplimentado por los 34 estudiantes de la muestra, los resultados obtenidos han sido los siguientes:

En la primera pregunta, en donde se preguntaba a los adolescentes por sí habían aumentado el peso durante el confinamiento, el 58,82\% de ellos (20) han contestado que su peso ha aumentado, mientras que 14 (el 41,17) han contesado que su peso no ha aumentado.

En la segunda pregunta, 32 alumnos (el 94,11\%) han indicado que se notan más nerviosos en relación antes del confinamiento, mientras que únicamente 2 estudiantes (el 5,88\%) no notan que se encuentren más nerviosos.

En la tercera pregunta, en relación al sueño, el 82,35\% (28 estudiantes) manifiestan que duermen peor ahora que antes de la pandemia, mientras que el 17,64\% (6 adolescentes) indican que no les ha afectado al sueño el confinamiento vivido.

En la cuarta pregunta, a la hora de concentrarse para realizar alguna actividad, el 76,47\% (26 estudiantes) indican que les cuesta más, mientras que el 23,52\% (8 alumnos), entienden que su capacidad de concentración es muy pareja a antes de la pandemia.

En la quinta y sexta pregunta se observa una clara similitud en la respuesta de los adolescentes, ya que el cien por cien de los encuestados manifiestan intranquilidad tanto a la hora de que sus familiares se puedan contagiar como a que personalmente ellos lo puedan hacer

\section{DISCUSIÓN}

Teniendo en cuenta los resultados alcanzados, se aprecia como los sujetos que han participado en la investigación muestran unas secuelas psicológicas derivadas de la pandemia que desde comienzos del año 2020 ha invadido el mundo.

Se observa como los adolescentes que han participado en esta investigación indican como a causa del confinamiento su peso ha aumentado, como se han observado en estudios anteriores como los de Balluerka, et. al., (2020). Como consecuencia del confinamiento sufrido durante gran parte del 2020 y siguiendo la línea de trabajo manifestada por Brooks et al. (2020), Ios adolescentes se sienten más nerviosos, afectando en su vida diaria y más concretamente en el sueño, donde indican que duermen peor como ocurre en anteriores investigaciones realizadas (Orgiles, et. Al, 2020)

Otro de los aspectos que se habían observado en investigaciones anteriores (Orgiles, et.al, 2020) es que la capacidad de concentración a la hora de realizar una actividad, había disminuido y en esta investigación se aprecia como también ocurre en un porcentaje alto de los estudiantes. Las preocupaciones que gracias a la pandemia afloran en el pensamiento de los adolescentes hacen que su capacidad de concentración en el momento de realizar una actividad se hayan visto afectadas

Donde se centra el mayor de las preocupaciones por parte de los adolescentes es a la hora pensar en que se puedan contagiar tanto ellos como las personas más cercanas. Todos los estudiantes de la muestran manifiestan esa preocupación, como ya se había preciado en estudios anteriores como los de Balluerka-Lasa et al. (2020) y Berasategui et al.(2020).

\section{CONCLUSIONES}

Teniendo en cuanta los resultados obtenidos en la investigación realizada y partiendo de los objetivos establecidos, las conclusiones alcanzadas son las siguientes:

Durante el confinamiento, una muestra representativa (el 58,82\%) manifiesta que han aumentado su peso. El 94,11\% de los encuestados han indicado que se sienten más nerviosos en relación a antes de la pandemia, afectando a su vida diaria y en concreto al suelo (el $82,35 \%$ indica que la calidad del sueño es peor que antes de la pandemia) y a su capacidad de concentración (el 76,47\% confiesa costarle más concentrarse). 


\section{LA INFLUENCIA FUTURA DE LA PANDEMIA DESDE EL PLANO PSICOLOGICO EN LOS ADOLESCENTES}

En relación a la pandemia, una de las mayores de sus preocupaciones es al temor de que se contagien tanto ellos como sus seres queridos. Todos los encuestados han manifestado esa preocupación.

En resumen, partiendo del objetivo general establecido en esta comunicación, donde era conocer la influencia de la pandemia desde el plano psicológico en los adolescentes, se observa como tras analizar varias variables, dicha pandemia está influyendo en el plano psicológico de los adolescentes, en concreto en el aumento de peso, en su estado emocional, debido a que se perciben más nerviosos, en la calidad del sueño, en su capacidad de concentración y en la preocupación por el posible contagio de sus seres queridos o de ellos en primera persona.

\section{LINEAS FUTURAS A TRABAJAR:}

Partiendo de las conclusiones indicadas, observando la influencia negativa que está teniendo la pandemia en los adolescentes, a través de esta comunicación se plantean las siguientes líneas de trabajo.

Realizar estudios empíricos con unas muestras elevadas, para conocer lo mejor posible la repercusión de la pandemia en la población adolescente.

Fundamentar los resultados empíricos que se obtengan con argumentos teóricos firmes y rigurosos.

Realizar programas didácticos para concienciar a la población de la importancia y consecuencias de la pandemia

\section{REFERENCIAS BIBLIOGRÁFICAS}

Balluerka Lasa N, Gómez Benito J, Hidalgo Montesinos MD, Gorostiaga Manterola A, Espada Sánchez JP, Padilla García JL \& Santed Germán MA (2020): Las consecuencias psicológicas de la COVID-19 y el confinamiento. Bilbao: Servicio de Publicaciones de la Universidad del País Vasco.

Berasategi Sancho, N. (Coord.), Idoiaga Mondragon, N., Dosil Santamaría, M., Picaza Gorrochategui, M., y Ozamiz Etxebarria, N. (2020). Las voces de los niños y de las niñas en situación de confinamiento por el COVID-19. Servicio de publicaciones de la Universidad de País Vasco. https://webargitalpena.adm.ehu.es/pdf/USP00202291.pdf

Brooks, S. K., Webster, R. K., Smith, L. E., Woodland, L., Wessely, S., Greenberg, N. y Rubin, G. J. (2020). The psychological impact of quarantine and how to reduce it: Rapid review of the evidence. The Lancet, 395(10227), 912-920. doi:10.1016/S0140-6736(20)30460-8

Espada, J. P., Orgilés, M., Piqueras, J. A. y Morales, A. (2020). Buenas prácticas en la atención psicológica infantojuvenil ante la COVID-19. Clínica y Salud. Avance online. doi:10.5093/clysa2020a14

Green P (2020). Risk to children and Young people during COVID-19 pandemic. BMJ, 28 abril; m1669

Oppedal, B., e Idsoe, T. (2012). Conduct Problems and Depression among Unaccompained Refugees: The Association with Pre-Migration Trauma and Acculturation. Anales de Psicología, 28 (3), 683-694. https://doi. org/10.6018/analesps.28.3.155981

Orgilés, M., Morales, A., Delveccio, E., Mazzesci, M., y Espada, J. P. (2020). Immediate psychological effects of the COVID-19 quarantine in youth from Italy and Spain. Accesible en: https://psyarxiv.com/5bpfz/

Real Decreto 463/2020, de 14 de marzo, por el que se declara el estado de alarma para la gestión de la situación de crisis sanitaria ocasionada por la COVID-19. Boletín Oficial del Estado, 14 de marzo de 2020, n.. 67 , pp. 25390-25400. Recuperado el 27/01/2020 https://www.boe.es/eli/es/rd/2020/03/14/463/con

Rodríguez-Hernández, P. J., Betancort, M., Ramírez-Santana, G. M., García, R., Sanz-Alvarez, E. J., y De las Cuevas-Castresana, C. (2014). Puntos de corte de la versión española del Cuestionario de Cualidades y Dificultades (SDQ). Revista de Psiquiatría Infanto-Juvenil, 31(3), 23-29. http://aepnya. eu/index.php/revistaaepnya/article/view/169

Sandín B, Valiente RM, García-Escalera J \& Chorot P. (2020) Impacto psicológico de la pandemia de COVID-19: Efectos negativosy positivos en población española asociados al periodode confinamiento nacional. Journal of Psychopathology and Clinical Psychology / Revista de Psicopatología y Psicología Clínica, 25 (1), 1-22. 
Doi: 10.5944/rppc.27569. http://www.aepcp.net. http://revistas.uned.es/index.php/rppc

Sandín, B., Chorot, P., Valiente, R. M., y Santed, M. A. (1998). Frecuencia e intensidad de los miedos en los niños: Datos normativos. Revista de Psicopatología y Psicología Clínica, 3(1),15-25. Recuperado de: https://www. aepcp.net/arc/1998_V3_N1_\%20pp.15-25.pdf

Upel (1998). El proyecto factible:Una modalidad de investigación. Recuperacdo de https://www.redalyc.org/pdf/410/41030203.pdf

Valiente, C., Vázquez, C., Peinado, V., Contreras, A., Trucharte, A., Bental, R., y Martínez, A. (2020). Síntomas de ansiedad, depresión y estrés postraumático ante el COVID-19: Prevalencia y predictores. UCM. https://www.ucm.es/ inventap/file/vida-covid19-informe-ejecutivomalestar3520 -final-1?ver

Valiente, R.M., Sandín, B., y Chorot, P. (2003). Miedos en la infancia y la adolescencia. UNED.

Wang, C., Pan, R., Wan, X., Tan, Y., Xu, L., Ho, C. S. y Ho, R. C. (2020). Immediate psychological responses and associated factors during the initial stage of the 2019 Coronavirus disease (COVID-19) epidemic among the general population in China. International Journal of Environmental Research and Public Health, 17(5), 1729. doi:10.3390/ijerph17051729 
